apuntesuniversitarios.upeu.edu.pe

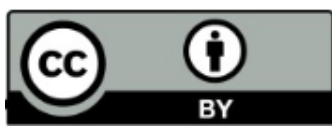

Apuntes Universitarios, 2020: 10(4), octubre-diciembre ISSN:

2304-0335 DOI:https://doi.org/10.17162/au.v10i4.491

\title{
Bienestar psicológico y resiliencia en estudiantes de educación superior pedagógica
}

\section{Psychological well-being and resilience in pedagogical higher education students}

\author{
Edwin Gustavo Estrada Araoz ${ }^{1}$ y Helen Juddy Mamani Uchasara ${ }^{2}$ \\ Universidad Nacional Amazónica de Madre de Dios, Perú ${ }^{12}$ \\ (iD) ORCID ID: https://orcid.org/0000-0003-4159-934X ${ }^{1}$ \\ (iD) ORCID ID: https://orcid.org/0000-0003-1735-8570²
}

Recibido: 07 de enero de 2020

Aceptado: 30 de mayo de 2020

\section{Resumen}

El objetivo de la presente investigación fue determinar la relación que existe entre el bienestar psicológico y la resiliencia en estudiantes del Instituto de Educación Superior Pedagógico Público "Nuestra Señora del Rosario" de Puerto Maldonado, 2019. El enfoque de investigación fue cuantitativo, el diseño no experimental y el nivel correlacional. La muestra fue conformada por 134 estudiantes y fue determinada mediante un muestreo probabilístico estratificado, los instrumentos de recolección de datos fueron: la Escala de Bienestar Psicológico de Sánchez Cánovas y la Escala de Resiliencia (ER) de Wagnild y Young. Los resultados determinaron que el bienestar psicológico se relaciona directa y significativamente con la resiliencia ya que el coeficiente de correlación rho de Spearman fue de 0,702 con un p-valor inferior al nivel de significancia $(\mathrm{p}=0,000<0,05)$. Finalmente, se concluye que mientras los estudiantes posean un adecuado bienestar psicológico, su capacidad de resiliencia será mayor y viceversa.

Palabras clave: Bienestar psicológico; resiliencia; perseverancia; educación superior; formación docente

\begin{abstract}
The objective of this research was to determine the relation between psychological well-being and resilience in students of the Institute of Public Pedagogical Higher Education "Nuestra Señora del Rosario" in the city of Puerto Maldonado, 2019. The research approach was quantitative, the design was not experimental and correlational level. The sample was made up of 134 students and was
\end{abstract}

${ }^{1}$ Correspondencia al autor

E-mail: edwin5721@outlook.com 
determined by a stratified probabilistic sampling, the data collection instruments were: the Sánchez Cánovas Psychological Welfare Scale and the Wagnild and Young Resilience Scale (ER). The results determined that psychological well-being was directly and significantly related to resilience since Spearman's rho correlation coefficient was 0.702 with a p-value below the level of significance $(p=0.000<0.05)$. Finally, it is concluded that as long as the students have adequate psychological well-being, their resilience will be greater and vice versa.

Keywords: Psychological well-being; resilience; perseverance; higher education; teacher training

\section{Introducción}

La demanda educativa en la etapa de educación superior en el Perú ha aumentado significativamente, la cual se ve reflejada en la tasa de matrícula bruta que aumentó en cerca de 30 puntos porcentuales entre el 2000 y 2014, situándose al $68.8 \%$ de la población de entre 15 y 64 años de edad (OCDE, 2016). Bajo esa perspectiva, y de acuerdo a Torres (2019), cerca de 1.9 millones de jóvenes entre 17 y 24 años cursan estudios de educación superior (universitaria, tecnológica o pedagógica), donde deben afrontar diferentes desafíos (personales, académicos y sociales) tales como ser cada vez más autónomos, socializar con más personas, aprobar satisfactoriamente los cursos, organizar su tiempo y tomar decisiones adecuadamente, lo cual influirá indudablemente en su bienestar psicológico (Sandoval, 2017).

Por otro lado, el bienestar psicológico ha sido y es un tema muy interesante para las diversas disciplinas y ramas de la psicología. Barrantes y Ureña (2015) sostienen que existe evidencia científica para afirmar que los estados de bienestar tienen una influencia directa en los diversos ámbitos de la vida; en ese sentido, el bienestar ha sido relacionado con variables como la felicidad, la salud mental así como la calidad de vida (Ballesteros et al., 2006); por otra parte, también ha sido asociado con los niveles de desempeño académico (Carranza et al., 2017; Osornio et al., 2011; Barrera et al., 2019; Trucchia et al., 2013).

De acuerdo con Ryan y Deci (2001), existen dos perspectivas desde las cuales se ha estudiado el bienestar psicológico durante las últimas décadas: la perspectiva hedónica, que sostiene que el bienestar está relacionado con el placer y la felicidad; vale decir, al balance entre las emociones placenteras y displacenteras y al grado de satisfacción con la vida (Cassaretto y Martínez, 2016); y la perspectiva eudaimónica, que se centra en el significado y la autorrealización y define el bienestar en términos del grado en que una persona está funcionando plenamente (Meléndez et al., 2018). A pesar de las diferencias expuestas, actualmente ambas perspectivas son consideradas no excluyentes (Keyes et al., 2002). 
Buscando un punto de equilibrio entre las dos perspectivas, Ryff (1989) propuso un modelo multidimensional, teniendo como base la perspectiva eudaimónica para explicar el bienestar psicológico; este se conforma por seis dimensiones: autoaceptación, relaciones positivas con otros, autonomía, dominio del entorno, propósito de vida y crecimiento personal. Asimismo, este autor afirma que "los estudios del bienestar psicológico centran el interés en el desarrollo personal de los individuos, en el estilo y manera de afrontar los retos vitales y en el esfuerzo y afán por conseguir metas” (p.2). De igual manera, Sandoval et al. (2017) complementan lo expuesto por Ryff (1989) señalando que:

El bienestar psicológico es una experiencia personal que se va construyendo a través del desarrollo psicológico de la persona y de la capacidad para relacionar y relacionarse de manera positiva e integrada con las experiencias de vida que va experimentando, considerando la incorporación activa de medidas positivas para ello y no solo la ausencia de los aspectos negativos que va vivenciando (p.3).

En ese sentido, un estudiante que presente niveles favorables de bienestar psicológico podrá desempeñarse adecuadamente en el contexto personal, familiar, académico y social. Por ello, es necesario que las instituciones formadoras-llámense universidades o institutos_promuevan su desarrollo.

En cuanto a la resiliencia, es definida por Luthar, Cicchetti y Becker (2000) como un proceso dinámico que conlleva a que la persona se adapte positivamente a situaciones adversas. "Los tres elementos clave de esta definición son los que configuran el concepto de resiliencia: el proceso, la adversidad y la adaptación positiva" (García et al., 2016, p.4). Esta definición parte de la observación de personas que pueden sobreponerse a contextos hostiles y adversos, lo cual motiva a que pongan en juego mecanismos de afrontamiento, adaptación y motivación para seguir adelante.

La resiliencia es una definición usualmente relacionada con las ciencias físicas y se refiere a la resistencia de los materiales así como a la capacidad de recuperarse al ser sometidos a diferentes fuerzas y presiones (García et al., 2016). Del mismo modo, Ortunio y Guevara (2016) afirman que en el campo de la psicología la resiliencia es un constructo bastante estudiado puesto que está relacionado con la salud mental y las estrategias de adaptación al ambiente por parte de las personas. Por ende, para que una persona desarrolle la resiliencia es importante el papel de la familia, la comunidad y la sociedad, puesto que son ellos quienes fomentan el surgimiento de los 
recursos personales, los cuales permiten la autogestión para su desarrollo tanto personal como social (García y Domínguez, 2013).

A la luz de las consideraciones expuestas, el objetivo de la presente investigación fue determinar la relación que existe entre el bienestar psicológico y la resiliencia en estudiantes del Instituto de Educación Superior Pedagógico Público "Nuestra Señora del Rosario”, en la ciudad de Puerto Maldonado, 2019.

\section{Metodología}

El enfoque de la presente investigación fue cuantitativo y el diseño no experimental, puesto que las variables no fueron manipuladas, sino se observaron los fenómenos tal como se dieron en su entorno y luego fueron analizados (Hernández, Fernández y Baptista, 2014). Asimismo, el nivel de investigación fue correlacional ya que se buscó conocer la relación que existe entre el bienestar psicológico y la resiliencia y ello se estimó a través del uso de coeficientes los cuales nos mostraron su intensidad y dirección (Bisquerra, 2009).

La población estuvo conformada por 205 estudiantes matriculados en el Instituto de Educación Superior Pedagógico Público “Nuestra Señora del Rosario” en el ciclo académico 2019 - II. Para hallar la muestra se realizó un muestreo probabilístico estratificado, quedando distribuida de la siguiente manera:

\section{Tabla 1}

Distribución de la muestra

\begin{tabular}{lcccc}
\hline \multicolumn{1}{c}{ Carreras profesionales } & Población & \% & Muestra & \% \\
\hline Educación Física & 46 & 22,44 & 30 & 22,44 \\
Educación Primaria & 52 & 25,37 & 34 & 25,37 \\
Educación Inicial & 84 & 40,98 & 55 & 40,98 \\
Educación Primaria Intercultural Bilingüe & 23 & 11,22 & 15 & 11,22 \\
Total & 205 & 100,00 & 134 & 100,00 \\
\hline
\end{tabular}

Fuente: Sistema Integrado de Gestión de la Educación Superior Pedagógica - SIGES

En el recojo de información sobre el bienestar psicológico se utilizó la Escala de Bienestar Psicológico de Sánchez Cánovas (2013). Consta de 65 ítems construidos en base a la escala de Likert (nunca, casi nunca, a veces, casi siempre y siempre) y estructurados en 4 dimensiones: Bienestar subjetivo, bienestar material, bienestar laboral y relaciones con la pareja. Las propiedades psicométricas de la escala fueron determinadas mediante el proceso de validación y confiabilidad. 
La validación de contenido se realizó a través de la técnica de juicio de expertos. El coeficiente V de Aiken fue de 0,837 lo que indica que el cuestionario tiene buena validez. Con relación a la confiabilidad, también fue determinada a través una prueba piloto, obteniéndose un coeficiente Alfa de Cronbach de 0,872 lo que quiere decir que el instrumento es altamente confiable.

Para evaluar la resiliencia se usó la Escala de Resiliencia (ER) de Wagnild y Young (1993), la cual se compone de 25 preguntas las cuales puntúan en una escala de tipo Likert de 7 puntos, donde 1 es en desacuerdo y un máximo de 7 que indica que el participante está muy de acuerdo. Presenta 5 dimensiones: confianza en sí mismo, ecuanimidad, perseverancia, satisfacción personal y sentirse bien solo. El coeficiente V de Aiken, determinado por los expertos, fue de 0,904 lo que indica que el cuestionario tiene muy buena validez y el índice Alfa de Cronbach fue de 0,889 lo que quiere decir que es confiable.

Para el análisis descriptivo se usaron las frecuencias y los porcentajes y para la prueba de hipótesis se recurrió a la prueba no paramétrica rho de Spearman. Esta prueba, además de contrastar las hipótesis, nos indicó la intensidad de las relaciones.

\section{Análisis de datos y discusión}

Luego de haber aplicado los instrumentos de recojo de datos se procedió a estructurar la base de datos y así valorar las puntuaciones para luego realizar las pruebas de hipótesis, tanto general como específicas. Los resultados que se exponen a continuación son resultado del tratamiento estadístico realizado a los datos obtenidos a través de los instrumentos de recolección de datos.

Tabla 2

Prueba de normalidad Kolmogorov - Smirnov para las variables y dimensiones de estudio

\begin{tabular}{lccc}
\hline & \multicolumn{3}{c}{ Kolmogorov-Smirnov $^{\mathrm{a}}$} \\
\cline { 2 - 4 } & Estadístico & $\mathrm{gl}$ & Sig. \\
\hline Bienestar psicológico &, 067 & 134 &, 200 \\
Resiliencia &, 088 & 134 &, 013 \\
Confianza en sí mismo &, 136 & 134 &, 000 \\
Ecuanimidad &, 121 & 134 &, 000 \\
Perseverancia &, 082 & 134 &, 028 \\
Satisfacción personal &, 120 & 134 &, 000 \\
Sentirse bien solo &, 150 & 134 &, 000 \\
\hline
\end{tabular}

a. Corrección de significación de Lilliefors 
En la tabla 2 se realizó la prueba de normalidad Kolmogorov - Smirnov donde podemos ver que las puntuaciones, tanto de las variables como de las dimensiones, no se ajustan a la distribución normal ( $\mathrm{p}<0,05)$. Por lo expuesto, viendo la naturaleza de las variables (categóricas), su nivel de medición (ordinal) así como la bondad de ajuste, se decidió utilizar la prueba no paramétrica rho de Spearman.

Tabla 3

Correlación entre el bienestar psicológico y la resiliencia

\begin{tabular}{|c|c|c|c|c|}
\hline & & & $\begin{array}{c}\text { Bienestar } \\
\text { psicológico }\end{array}$ & Resiliencia \\
\hline \multirow{6}{*}{$\begin{array}{c}\text { Rho de } \\
\text { Spearman }\end{array}$} & \multirow{3}{*}{$\begin{array}{c}\text { Bienestar } \\
\text { psicológico }\end{array}$} & Coeficiente de correlación & 1,000 &, $702^{* *}$ \\
\hline & & Sig. (bilateral) & ent &, 000 \\
\hline & & $\mathrm{N}$ & 134 & 134 \\
\hline & \multirow{3}{*}{ Resiliencia } & Coeficiente de correlación & ,702** & 1,000 \\
\hline & & Sig. (bilateral) & ,000 & • \\
\hline & & $\mathrm{N}$ & 134 & 134 \\
\hline
\end{tabular}

**. La correlación es significativa en el nivel 0,01 (2 colas).

De acuerdo a la tabla 3, el coeficiente de correlación rho de Spearman entre las variables bienestar psicológico y resiliencia es de 0,702 con un p-valor inferior al nivel de significancia $(\mathrm{p}=0,000<0,05)$, por lo tanto, se rechaza la hipótesis nula y se acepta la hipótesis alterna, la cual indica que existe una relación alta, directa y significativa entre ambas variables. En virtud a los resultados expuestos se establece que mientras los estudiantes presenten un alto nivel de bienestar psicológico, presentarán una mayor capacidad de resiliencia frente a las dificultades que se les presenten en la vida.

Tabla 4

Correlación entre el bienestar psicológico y la confianza en sí mismo

\begin{tabular}{|c|c|c|c|c|}
\hline & & & $\begin{array}{c}\text { Bienestar } \\
\text { psicológico }\end{array}$ & $\begin{array}{c}\text { Confianza en sí } \\
\text { mismo } \\
\end{array}$ \\
\hline \multirow{2}{*}{$\begin{array}{c}\text { Rho de } \\
\text { Spearman }\end{array}$} & $\begin{array}{l}\text { Bienestar } \\
\text { psicológico }\end{array}$ & $\begin{array}{l}\text { Coeficiente de correlación } \\
\text { Sig. (bilateral) } \\
\mathrm{N}\end{array}$ & $\begin{array}{c}1,000 \\
134\end{array}$ & $\begin{array}{c}, 684^{* *} \\
, 000 \\
134\end{array}$ \\
\hline & $\begin{array}{c}\text { Confianza en sí } \\
\text { mismo }\end{array}$ & $\begin{array}{l}\text { Coeficiente de correlación } \\
\text { Sig. (bilateral) } \\
\text { N }\end{array}$ & $\begin{array}{l}, 684^{* *} \\
, 000 \\
134\end{array}$ & $\begin{array}{c}1,000 \\
\dot{134}\end{array}$ \\
\hline
\end{tabular}

**. La correlación es significativa en el nivel 0,01 (2 colas). 
Como se puede observar en la tabla 4, el coeficiente de correlación rho de Spearman entre la variable bienestar psicológico y la dimensión confianza en sí mismo es de 0,684 con un p-valor inferior al nivel de significancia $(\mathrm{p}=0,000<0,05)$; por ello, se rechaza la hipótesis nula y se acepta la hipótesis alterna, la cual señala que existe una relación alta, directa y significativa entre la variable y dimensión antes mencionada. En ese sentido, se puede indicar que si los estudiantes presentan altos niveles de bienestar psicológico, tendrán una mayor confianza en sí mismo al momento de desenvolverse personal, laboral o académicamente.

\section{Tabla 5}

Correlación entre el bienestar psicológico y ecuanimidad

\begin{tabular}{cclcc}
\hline & & & $\begin{array}{c}\text { Bienestar } \\
\text { psicológico }\end{array}$ & Ecuanimidad \\
\hline \multirow{3}{*}{ Rho de } & Bienestar & Coeficiente de correlación & 1,000 &, $550^{* *}$ \\
psicológico & Sig. (bilateral) & $\mathrm{N}$ & 134 &, 000 \\
& \multirow{2}{*}{ Ecuarman } & Coeficiente de correlación &, $550^{* *}$ & 134 \\
\cline { 2 - 5 } & \multirow{2}{*}{ Ecuanimidad } & Sig. (bilateral) &, 000 & 1,000 \\
& & $\mathrm{~N}$ & 134 & 134 \\
\hline
\end{tabular}

**. La correlación es significativa en el nivel 0,01 (2 colas).

Según la tabla 5, el coeficiente de correlación rho de Spearman entre la variable bienestar psicológico y la dimensión ecuanimidad es de 0,550 con un p-valor inferior al nivel de significancia ( $\mathrm{p}=0,000<0,05)$; por lo tanto, se rechaza la hipótesis nula y se acepta la hipótesis alterna, la cual indica que existe una relación moderada, directa y significativa entre la variable y dimensión antes mencionada. Desde esta perspectiva, los estudiantes que mantengan niveles elevados de bienestar psicológico tendrán la ecuanimidad para afrontar situaciones adversar en los diferentes ámbitos de su vida.

\section{Tabla 6}

Correlación entre el bienestar psicológico y perseverancia

\begin{tabular}{|c|c|c|c|c|}
\hline & & & $\begin{array}{c}\text { Bienestar } \\
\text { psicológico }\end{array}$ & Perseverancia \\
\hline \multirow{2}{*}{$\begin{array}{l}\text { Rho de } \\
\text { Spearman }\end{array}$} & $\begin{array}{l}\text { Bienestar } \\
\text { psicológico }\end{array}$ & $\begin{array}{l}\text { Coeficiente de correlación } \\
\text { Sig. (bilateral) } \\
\text { N }\end{array}$ & $\begin{array}{c}1,000 \\
134 \\
\end{array}$ & $\begin{array}{c}, 526^{* *} \\
, 000 \\
134\end{array}$ \\
\hline & Perseverancia & $\begin{array}{l}\text { Coeficiente de correlación } \\
\text { Sig. (bilateral) } \\
\mathrm{N}\end{array}$ & $\begin{array}{l}526^{* *} \\
, 000 \\
134 \\
\end{array}$ & $\begin{array}{c}1,000 \\
134 \\
\end{array}$ \\
\hline
\end{tabular}

**. La correlación es significativa en el nivel 0,01 (2 colas). 
De acuerdo a la tabla 6, el coeficiente de correlación rho de Spearman entre la variable bienestar psicológico y la dimensión perseverancia es de 0,526 con un p-valor inferior al nivel de significancia $(\mathrm{p}=0,000<0,05)$; en virtud a ello, se rechaza la hipótesis nula y se acepta la hipótesis alterna, la cual señala que existe una relación moderada, directa y significativa entre la variable y dimensión antes mencionada. En ese sentido, se infiere que cuando un estudiante presenta un bienestar psicológico, actúa con perseverancia hasta alcanzar los objetivos que se propuso.

\section{Tabla 7}

Correlación entre el bienestar psicológico y satisfacción personal

\begin{tabular}{|c|c|c|c|c|}
\hline & & & $\begin{array}{c}\text { Bienestar } \\
\text { psicológico }\end{array}$ & $\begin{array}{c}\text { Satisfacción } \\
\text { personal }\end{array}$ \\
\hline \multirow{6}{*}{$\begin{array}{c}\text { Rho de } \\
\text { Spearman }\end{array}$} & \multirow{3}{*}{$\begin{array}{c}\text { Bienestar } \\
\text { psicológico }\end{array}$} & Coeficiente de correlación & 1,000 &, $411^{* *}$ \\
\hline & & Sig. (bilateral) & & ,000 \\
\hline & & $\mathrm{N}$ & 134 & 134 \\
\hline & \multirow{3}{*}{$\begin{array}{c}\text { Satisfacción } \\
\text { personal }\end{array}$} & Coeficiente de correlación & $411^{* *}$ & 1,000 \\
\hline & & Sig. (bilateral) & 000 & t \\
\hline & & $\mathrm{N}$ & 134 & 134 \\
\hline
\end{tabular}

**. La correlación es significativa en el nivel 0,01 (2 colas).

Según la tabla 7, el coeficiente de correlación rho de Spearman entre la variable bienestar psicológico y la dimensión satisfacción personal es de 0,411 con un p-valor inferior al nivel de significancia $(\mathrm{p}=0,000<0,05)$; por ello, se rechazó la hipótesis nula y aceptó la hipótesis alterna, la cual indica que existe una relación moderada, directa y significativa entre la variable y dimensión antes mencionada. Ello implica que una persona que alcance un bienestar psicológico tendrá una mirada positiva sobre las cosas que está haciendo.

\section{Tabla 8}

Correlación entre el bienestar psicológico y sentirse bien solo

\begin{tabular}{|c|c|c|c|c|}
\hline & & & $\begin{array}{l}\text { Bienestar } \\
\text { psicológico }\end{array}$ & $\begin{array}{l}\text { Sentirse bien } \\
\text { solo }\end{array}$ \\
\hline \multirow{6}{*}{$\begin{array}{c}\text { Rho de } \\
\text { Spearman }\end{array}$} & \multirow{3}{*}{$\begin{array}{l}\text { Bienestar } \\
\text { psicológico }\end{array}$} & Coeficiente de correlación & 1,000 &, $546^{* *}$ \\
\hline & & Sig. (bilateral) & s. &, 000 \\
\hline & & $\mathrm{N}$ & 134 & 134 \\
\hline & \multirow{3}{*}{ Sentirse bien solo } & Coeficiente de correlación &, $546^{* *}$ & 1,000 \\
\hline & & Sig. (bilateral) & ,000 & • \\
\hline & & $\mathrm{N}$ & 134 & 134 \\
\hline
\end{tabular}

**. La correlación es significativa en el nivel 0,01 (2 colas). 
Finalmente, en la tabla 8 se puede observar que el coeficiente de correlación rho de Spearman entre la variable bienestar psicológico y la dimensión sentirse bien solo es de 0,546 con un $\mathrm{p}$-valor inferior al nivel de significancia $(\mathrm{p}=0,000<0,05)$; por ello, se rechaza la hipótesis nula y se acepta la hipótesis alterna, la cual señala que existe una relación moderada, directa y significativa entre la variable y dimensión en estudio. En ese sentido, se puede afirmar que cuando los estudiantes alcanzan un bienestar psicológico, se valoran como personas únicas e importantes, capaces de poder enfrentar las situaciones que se les presenten.

\section{Discusión}

El objetivo de la presente investigación fue determinar la relación que existe entre el bienestar psicológico y la resiliencia, lo cual fue demostrado mediante el coeficiente de correlación rho de Spearman $(r s=702 ; \mathrm{p}=0,000<0,05)$. Ello significaría que, mientras los estudiantes perciban un alto nivel de bienestar psicológico, presentarán una mayor capacidad de resiliencia frente a las dificultades que se les presenten en la vida. Este resultado guarda relación con los hallazgos de diversas investigaciones (Hernández, 2015; Piña, 2017; Benavente y Quevedo, 2018; Parada, 2015; Giraldo, 2013; Rosario, 2014; Morales y Gonzáles, 2014; Bustamante, 2019) quienes encontraron la existencia de relaciones directas entre las variables analizadas.

Con relación al primer objetivo específico, se demostró que el bienestar psicológico se relaciona significativamente con la dimensión confianza en sí mismo ( $r s=684 ; p=0,000<0,05)$ lo cual implicaría que las personas que tengan una valoración positiva de sus logros y una satisfacción personal con las acciones pasadas, presentes o futuras (Velásquez et al., 2008), tendrán una mayor capacidad de recuperarse, sobreponerse y adaptarse con éxito frente a la adversidad (Morales y Gonzáles, 2014). Este resultado es corroborado por Benavente y Quevedo (2018) quien pudo establecer que, al aumentar la confianza en uno mismo, las distintas dimensiones del bienestar psicológico también aumentan y por el contrario, al disminuir la confianza en uno mismo disminuyen dichas dimensiones. Según los datos presentados se puede afirmar que la confianza en uno mismo es la dimensión que se relaciona más con el bienestar psicológico.

Con relación al segundo objetivo específico, se halló que existe relación significativa entre el bienestar psicológico y la ecuanimidad $(r s=550 ; \mathrm{p}=0,000<0,05)$ lo que significaría que cuando las personas perciben de mejor manera su bienestar psicológico, toman las cosas con tranquilidad y moderan sus actitudes al enfrentar diferentes adversidades. Este hallazgo corrobora la investigación realizada por Benavente y Quevedo (2018) quienes establecieron que existe relación 
entre las escalas del bienestar psicológico y la ecuanimidad que demuestren para afrontar los retos que se les presenten.

En cuanto al tercer objetivo específico, se pudo hallar que existe relación significativa entre el bienestar psicológico y la perseverancia ( $r s=526 ; \mathrm{p}=0,000<0,05$ ), lo cual supone que si una persona presenta un adecuado bienestar psicológico, tendrá altos niveles de logro y autodisciplina, lo que le permitirá persistir ante la adversidad o desaliento y viceversa. Este resultado refuerza el hallazgo de Guil et al. (2016) quien estableció que existe relación significativa entre 3 dimensiones del bienestar psicológico (autoaceptación, autonomía y propósito de vida) y la perseverancia.

Respecto al cuarto objetivo específico, se logró establecer que existe relación significativa entre la variable bienestar psicológico y la dimensión satisfacción personal ( $r s=526$; $\mathrm{p}=0,000<0,05)$, lo que indica que las personas que presenten un alto nivel de bienestar psicológico tendrían una mirada positiva sobre las cosas que está haciendo y sentirán que, mediante sus acciones, están contribuyendo a darle un significado a su vida. Estos resultados van de la mano con los hallazgos de Guil et al. (2016) quien pudo determinar que todas las escalas del bienestar psicológico se correlacionan significativamente con la satisfacción personal. Por último, se pudo demostrar que la variable bienestar psicológico se relaciona significativamente con la dimensión sentirse bien solo $(r s=546 ; \mathrm{p}=0,000<0,05)$ lo que significaría que una persona que percibe que ha alcanzado el bienestar psicológico se valorará como alguien único e importante, capaz de poder enfrentar las situaciones que se les presenten. Estos resultados coinciden con los hallazgos de Gonzáles (2017) quien estableció que existe correlación significativa entre las dimensiones del bienestar psicológico (aceptación, autonomía, vínculos y proyectos) y la dimensión sentirse bien solo.

Los hallazgos encontrados se limitan al presente grupo de estudiantes, por lo que sería importante que las futuras investigaciones incrementen la muestra de estudio a otros grupos para poder confirmar los resultados que fueron obtenidos y ampliar la generalización de los mismos. Por otra parte, sería relevante que también incorporen y asocien las variables intervinientes como la edad, el sexo, la composición familiar y el nivel socioeconómico de los estudiantes para determinar los posibles factores de riesgo.

\section{Conclusión}

Los hallazgos del presente estudio muestran que existe correlación directa y significativa entre el bienestar psicológico y la resiliencia así como sus subescalas. Los coeficientes oscilan entre 
los niveles alto y moderado, lo cual indica que el bienestar psicológico es una de las principales variables que se asocian a la resiliencia. A la luz de los resultados, es menester que el instituto diseñe programas preventivos, a través del Área de Consejería y Tutoría, que contribuyan con el bienestar psicológico de todos los estudiantes para que así se caractericen por ser altamente resilientes.

\section{Referencias}

Ballesteros, P., Medina, A. y Caycedo, C. (2006). El bienestar psicológico definido por asistentes a un servicio de consulta psicológica en Bogotá, Colombia. Universitas Psychologica, 5(2), 239-258. Recuperado de http://www.scielo.org.co/pdf/rups/v5n2/v5n2a04.pdf

Barrantes, K. y Ureña, P. (2006). Bienestar psicológico y bienestar subjetivo en estudiantes universitarios costarricenses. Revista Intercontinental de Psicología y Educación, 17(1), 101123. Recuperado de https://www.redalyc.org/pdf/802/80242935006.pdf

Barrera, L., Sotelo, M., Barrera, R. y Aceves, J. (2019). Bienestar psicológico y rendimiento académico en estudiantes universitarios. Enseñanza e Investigación en Psicología, 1(2), 244251. Recuperado de https://revistacneip.org/index.php/cneip/article/view/42/33

Benavente, M. y Quevedo, M. (2018). Resiliencia, bienestar psicológico y afrontamiento en universitarios atendiendo a variables de personalidad y enfermedad. Revista de Psicología y Educación, 13(2), 99-112. https://doi.org/10.23923/rpye2018.01.161

Bisquerra, R. (2009). Metodología de la investigación educativa. (2a ed.). Madrid: La Muralla S.A. Carranza, R., Hernández, R. y Alhuay, J. (2017). Bienestar psicológico y rendimiento académico en estudiantes de pregrado de psicología. Revista Internacional de Investigación en Ciencias Sociales, 13(2), 133-146. Recuperado de http://scielo.iics.una.py/pdf/riics/v13n2/22264000-riics-13-02-00133.pdf

Cassaretto, M. y Martínez, P. (2017). Validación de las escalas de bienestar, de florecimiento y afectividad. Pensamiento Psicológico, 15(1), 19-31. Recuperado de https://www.redalyc.org/pdf/801/80149351002.pdf

García, J., García del Castillo, A., López, C. y Días, P. (2016). Conceptualización teórica de la resiliencia psicosocial y su relación con la salud. Salud y Drogas, 16(1), 59-68. Recuperado de https://www.redalyc.org/pdf/839/83943611006.pdf

García, M. y Domínguez, E. (2013). Desarrollo teórico de la Resiliencia y su aplicación en situaciones adversas: Una revisión analítica. Revista Latinoamericana de Ciencias Sociales, 
Niñez $\quad y \quad$ Juventud, $11(1), \quad$ 63-77. $\quad$ Recuperado de https://www.redalyc.org/pdf/773/77325885001.pdf

Giraldo, M. (2013). Resiliencia y Bienestar Psicológico según la duración del desempleo en una muestra de profesionales colombianos. Revista Interamericana de Psicología Ocupacional, 32(2), 22-37. Recuperado de http://revista.cincel.com.co/index.php/RPO/article/view/145/145

Gonzáles, J. (2017). Resiliencia y bienestar psicológico en madres de hijos con trastorno del espectro autista de un Centro de Educación Básica Especial de Lima Sur. (Tesis de Licenciatura). Universidad Autónoma del Perú, Lima, Perú. Recuperado de: http://repositorio.autonoma.edu.pe/bitstream/AUTONOMA/431/1/JEAMPIERT\%20FELIP E\%20GONZALES\%20BERRU.pdf

Guil, R., Zayas, A., Gil, P., Guerrero, C., Gonzáles, S. y Mestre, J. (2016). Bienestar psicológico, optimismo y resiliencia en mujeres con cáncer de mama. Psicooncología, 13(1), 127-138. https://doi.org/10.5209/rev_PSIC.2016.v13.n1.52492

Hernández, R. (2015). Resiliencia y bienestar psicológico de un grupo de adolescentes en riesgo de exclusión social. (Tesis de Licenciatura). Universidad de Salamanca, Salamanca, España. Recuperado https://gredos.usal.es/bitstream/handle/10366/126752/TFG_HernandezEstebanR_Resilienci aybienestar.pdf;jsessionid=79E164BE04B44FE623A4FE65FA250542? sequence=1

Hernández, R. Fernández, C. y Baptista, P. (2014) Metodología de la investigación. (6ªed.). México: Mc Graw-Hill.

Keyes, C., Shmotkin, D. y Ryff, C. (2002). Optimizing Well-Being: The Empirical Encounter of Two Traditions. Journal of Personality and Social Psychology, 82(6), 1007-1022. Recuperado de http://aging.wisc.edu/pdfs/871.pdf

Luthar, S., Cicchetti, D. y Becker, B. (2000). The construct of resilience: a critical evaluation and guidelines for future work. Child development, 71(3), 543-562. Recuperado de https://www.ncbi.nlm.nih.gov/pmc/articles/PMC1885202/\#

Meléndez, J., Agustí, A., Delhom, I., Reyes, M. y Satorres, E. (2018). Bienestar subjetivo y psicológico: comparación de jóvenes y adultos mayores. Summa Psicológica UST, 15(1), 18 24. Recuperado de https://summapsicologica.cl/index.php/summa/article/view/335/354 
Morales, M. y Gonzáles, A. (2014). Resiliencia-Autoestima-Bienestar psicológico y Capacidad intelectual de estudiantes de cuarto medio de buen rendimiento de liceos vulnerables. Estudios Pedagógicos, 40(1), 215-228. Recuperado de: https://scielo.conicyt.cl/pdf/estped/v40n1/art13.pdf

Muratori, M., Zubieta, E., Ubillos, S. y Gonzáles, J. (2015). Felicidad y Bienestar Psicológico: Estudio Comparativo Entre Argentina y España. Psykhe, 24(2), 1-18. Recuperado de http://www.psykhe.cl/index.php/psykhe/article/view/900/pdf_60

Organization for Economic Cooperation and Development (2016). Avanzando hacia una mejor educación para Perú. Lima: OCDE. Recuperado de https://www.oecd.org/dev/Avanzandohacia-una-mejor-educacion-en-Peru.pdf

Ortunio C, Magaly S, \& Guevara R, Harold. (2016). Aproximación teórica al constructo resiliencia. Comunidad $y \quad$ Salud, $14(2), \quad 96-105 . \quad$ Recuperado de http://ve.scielo.org/pdf/cs/v14n2/art12.pdf

Osornio, L., Sánchez de Tagle, R., Heshiki, L. y Valadez, S. (2011). El bienestar psicológico, predictor del rendimiento académico en estudiantes de la carrera de médico cirujano. Archivos en Medicina Familiar, 13(3), 111-116. Recuperado de https://www.redalyc.org/pdf/507/50730960002.pdf

Parada, P. (2015). Resiliencia, bienestar psicológico y autoeficacia en relación con el estrés en el docente joven y las prácticas encaminadas a la prevención de la violencia escolar (Tesis de Licenciatura). Universidad Piloto de Colombia, Bogotá, Colombia. Recuperado de http://polux.unipiloto.edu.co:8080/00002511.pdf

Piña, K. (2017). Bienestar psicológico y resiliencia en jóvenes universitarios (Tesis de Maestría). Universidad Autónoma del Estado de México, Toluca, México. Recuperado de http://hdl.handle.net/20.500.11799/68666

Rosario, F. (2014). Bienestar psicológico, resiliencia y rendimiento académico en estudiantes universitarios de primer ciclo de una Universidad Privada de Lima Metropolitana (Tesis de Maestría). Universidad Peruana Cayetano Heredia, Lima, Perú. Recuperado de http://repositorio.upch.edu.pe/handle/upch/1076

Ryan, R. y Deci, E. (2001). On Happiness and Human Potentials: A Review of Research on Hedonic and Eudaimonic Well-Being. Annual Review of Psychology, 52, 141-166. 
Ryff, C. (1989). Happiness is not everything or is it? Explorations on the meaning of psychological well-being. Journal of Personality and Social psychology, 57, 1069-1089.

Sánchez-Cánovas, J. (2013). Escala de bienestar psicológico. (3 ${ }^{\mathrm{a}}$ Ed.). Madrid: TEA Ediciones.

Sandoval, S., Dorner, A. y Véliz, A. (2017). Bienestar psicológico en estudiantes de carreras de la salud. Investigación en Educación Médica, 6(24), 260-266. Recuperado de http://riem.facmed.unam.mx/sites/all/archivos/A6Num24/08_AO_Bienestar.pdf

Torres, G. (2019). Gestión educativa y calidad de la educación en la Universidad Científica del Sur - cursos básicos, 2018-1. (Tesis de Maestría). Universidad de San Martín de Porres, Lima, $\quad$ Perú. $\quad$ Recuperado de http://www.repositorioacademico.usmp.edu.pe/bitstream/handle/usmp/5374/torres_vgn.pdf ? sequence $=1$

Trucchia, S., Lucchese, M., Enders, J. y Fernández, R. (2013). Relación entre rendimiento académico, bienestar psicológico y estrategias de afrontamiento en estudiantes de la carrera de medicina. Revista de la Facultad de Ciencias Médicas, 70(2), 144-152. Recuperado de https://revistas.unc.edu.ar/index.php/med/article/view/20189/19836

Velásquez, C., Montgomery, W., Montero, V., Pomalaya, R., Dioses, A., Velásquez, N., Araki, R. y Reynoso, D. (2008). Bienestar psicológico, asertividad y rendimiento académico en estudiantes universitarios sanmarquinos. Revista IIPSI, 11(2), 139-152. Recuperado de: https://revistasinvestigacion.unmsm.edu.pe/index.php/psico/article/view/3845/3078

Wagnild, G. y Young, H. (1993) Development and psychometric evaluation of the Resilience Scale. Journal of Nursing Measurement. 1(2), 165-177. 